\title{
Impacto de la infección nosocomial en un hospital de Bogotá, Colombia: efectos en mortalidad y costos
}

\author{
Carlos Humberto Saavedra, Karen Melissa Ordóñez y Jorge Augusto Díaz
}

\section{Nosocomial infections impact in a hospital in Bogota, Colombia: effects on mortality and hospital costs}

Background: Nosocomial infections (NI) are events associated with high impact on hospital costs and mortality. Aim: To evaluate from the health provider's perspective the costs and mortality attributable to NI. Methods: We selected a sample of patients with and without NI matched by age and diagnosis at admission. Costs were calculated and converted from Colombian pesos to US dollars using the average exchange rate of 2008. We evaluated the mortality rate in both groups. Results: We collected data on 187 patients with NI and 276 without NI. Median total hospitalization cost was US\$ 6,329 (95\% CI US\$5,527-7,934) in NI patients, while in non-infected patients this median was US\$1,207 (95\% CI US\$ 974-1,495). Mortality was higher in the NI group (31.6\% versus 5.1\%). Patients with NI had longer hospital stays (median 21 days, 95\% CI 18-24 days) than non-infected patients (median 5 days, 95\% CI 5-6 days). Mortality was also markedly higher in the NI group than in the non-infected group (31.6\% versus 5.1\%). Conclusion: NI are adverse and costly events related to patient attention that affect adversely the quality of attention.

Key words: Cost analysis, mortality, cross infection.

Palabras clave: Análisis de costos, mortalidad, infección hospitalaria.

\section{Introducción}

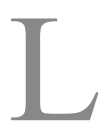

as infecciones asociadas a la atención en salud (IAAS) son eventos adversos prevenibles. Diferentes estudios han demostrado que las IAAS son causa de un incremento en los días de hospitalización, mayor uso de ayudas diagnósticas, consumo de medicamentos y riesgo de mortalidad $^{1,2}$.

Los estudios publicados han evaluado principalmente el costo y mortalidad atribuible a infecciones asociadas a dispositivos invasores en el contexto de la unidad de cuidado intensivo (UCI), lo cual no permite evaluar el impacto total de las IAAS ya que una gran proporción de éstas se presenta en salas generales y no siempre asociadas a dispositivos ${ }^{3-6}$. El conocimiento del impacto de las IAAS en general así como por tipo, es útil, especialmente en nuestro medio donde los recursos para la salud son limitados, para optimizar las intervenciones dirigidas a su prevención.

Dado el vacío del conocimiento del impacto de las IAAS en nuestro país, se consideró determinar el costo y la mortalidad atribuible a todos los tipos de IAAS, así como los costos diferenciales por tipo de IAAS.

\section{Métodos}

Este estudio fue realizado en el Hospital Universitario Clínica San Rafael, institución de carácter privado, el cual cuenta con 395 camas y cinco UCI (UCI coronaria, UCI médico-quirúrgica, UCI cardiovascular, UCI pediátrica, UCI neonatal).

Se realizó un diseño de casos y controles. El cálculo del tamaño de la muestra se realizó a partir de la mortalidad atribuible a IAAS. Para obtener un poder del 90\% se calculó un tamaño de muestra de 196 pacientes con IAAS y 196 sin IAAS. Para alcanzar el tamaño de la muestra, se incluyó la totalidad de pacientes diagnosticados con IAAS del año 2008 de acuerdo a las definiciones de los CDC (Centers for Diseases Control and Prevention, 2008, (7) ). La selección de los controles se realizó de forma pareada por diagnóstico de ingreso, edad, patologías concomitantes, gravedad e intervenciones.

Los pacientes remitidos de otras instituciones fueron excluidos del estudio. A los pacientes seleccionados, se les registró información demográfica, diagnóstico principal de ingreso, patologías asociadas, fecha de ingreso y egreso de la institución, requerimiento de UCI, tipo de IAAS presentada, número de IAAS presentadas, aislamiento microbiológico y estado al egreso.

Los costos fueron medidos en pesos colombianos del año 2008 y convertidos a dólares según la tasa de cambio del 04 de septiembre de 2008 ( 1 dólar $=1.992,59$ pesos colombiano). Se utilizó el método de costo directo, con cálculo de costos fijos y variables según la perspectiva del prestador. Como costos variables se definieron: el costo día-cama (US\$ 82,2), enfermería (asignación de enferme-

\author{
Hospital Universitario Clínica \\ San Rafael, Bogotá, \\ Colombia (CHS). \\ Universidad Nacional \\ de Colombia, Bogotá, \\ Colombia (CHS, KMO, JAD). \\ Los autores declaran no presentar \\ conflicto de interés. \\ El estudio fue realizado con el \\ presupuesto aportado por la \\ división de investigación de la \\ Universidad Nacional de Colombia. \\ Código DIB: 201010014671 \\ Recibido: 23 de mayo de 2014 \\ Aceptado: 5 de noviembre de 2014
}

Correspondencia a: Karen Melissa Ordóñez Díaz. karenmelissao@gmail.com 


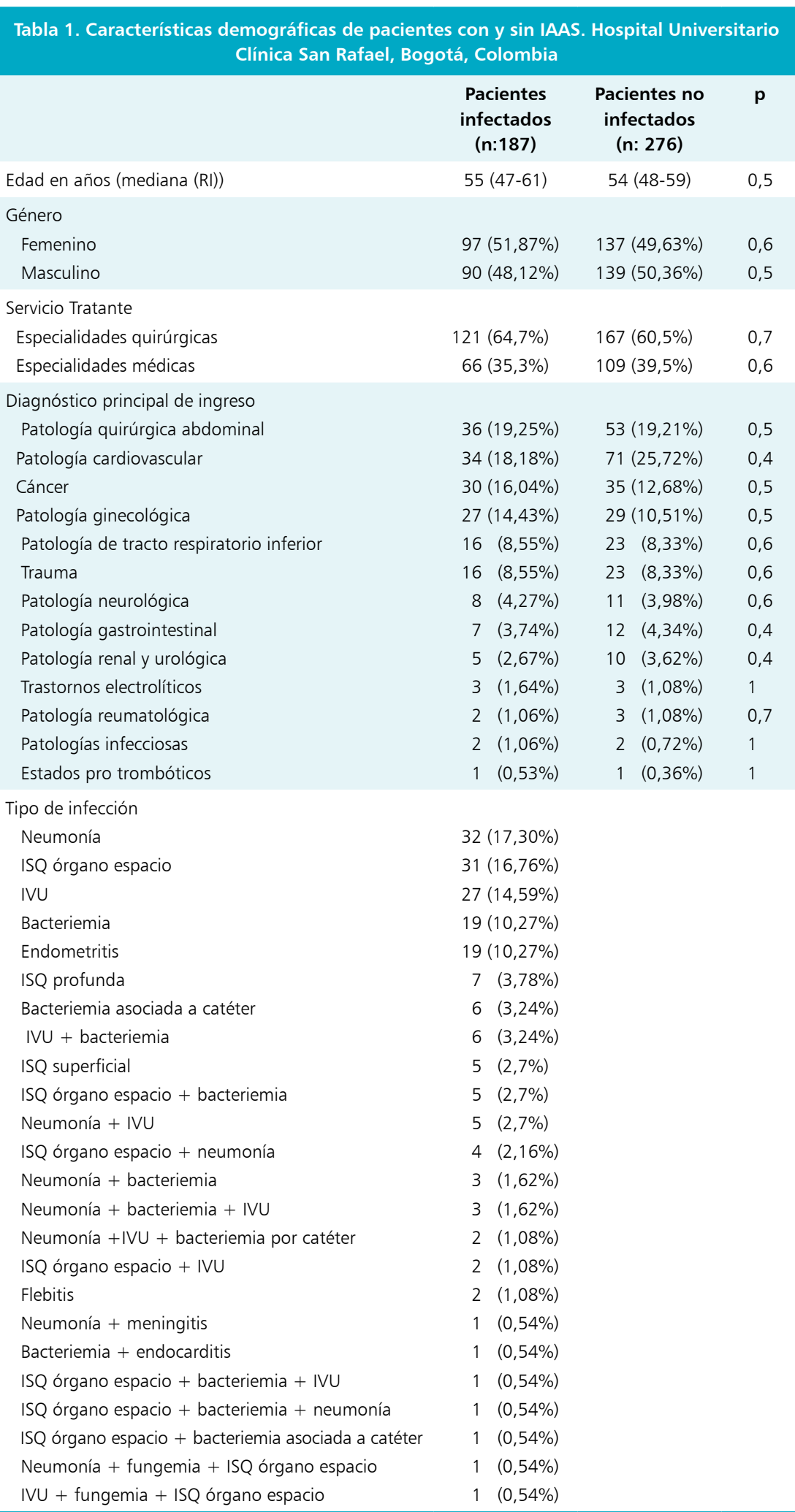

IAAS: infección asociada a la atención en salud. RI: rango intercuartílico. ISQ; infección del sitio quirúrgico. IVU: ifección de vías urinarias. ra exclusiva para pacientes aislados), valoración médica diaria, laboratorios, imágenes diagnósticas, interconsultas por especialista y medicamentos. Adicionalmente, se discriminó el costo de la hospitalización en UCI, el costo de antimicrobianos y el de otros medicamentos para servicios UCI y no UCI. Los costos de administración fueron considerados fijos (secretaria, auditoría, computador, dirección, mensajería). Los costos de los servicios prestados fueron de acuerdo al manual tarifario SOAT 2008.

Los costos derivados de las complicaciones presentadas durante la hospitalización fueron asumidos por el pagador.

El análisis de costos no se realizó desde la perspectiva de la sociedad, por lo que no se incluyó en éste el costo de incapacidades laborales, años de vida perdidos ni demandas judiciales.

El análisis de las variables de costos, estancia y mortalidad se realizó con estadística no paramétrica, dado que la distribución de las variables continuas no fue normal. El valor atribuible a la IAAS se obtuvo de la diferencia entre las medianas de los costos de pacientes con IAAS y sin IAAS. La información se llevó a una base plana (Excel 2007 y se analizó con el paquete estadístico Stata 9.0 (StataCorp, College Station, TX).

\section{Resultados}

Se incluyeron 187 pacientes con 233 IAAS y 276 pacientes sin IAAS. Los pacientes presentaron 91 diagnósticos de ingreso diferentes. Para el análisis estadístico se agruparon los diagnósticos en 13 grupos según el tipo de patología y la similitud de procedimientos médicoquirúrgicos realizados. Las características demográficas se describen en la Tabla 1.

Los servicios que más aportaron pacientes con IAAS fueron los pertenecientes a las especialidades quirúrgicas $(63,6 \%)$, en comparación a los de las especialidades médicas $(36,4 \%)$. Los tipos de infección más frecuentes fueron: neumonía (17,3\%), infección del sitio quirúrgico (ISQ) órgano espacio $(16,8 \%)$ e infección de vías urinarias $(14,6 \%)$. La presentación de más de una IAAS durante el mismo período de hospitalización se encontró en 37 $(19,1 \%)$ de los pacientes. La identificación del agente microbiológico se obtuvo en $57,2 \%$ de los casos. Los microorganismos aislados con mayor frecuencia fueron bacilos gramnegativos $(78,5 \%)$, seguidos de cocáceas grampositivas $(18,7 \%)$ y hongos $(8,4 \%)$. En $16,8 \%$ de los pacientes con aislamiento microbiológico, se documentó infección por más de un microorganismo. Los desenlaces de los pacientes con y sin IAAS se muestran en las Tablas 2 y 3.

Los pacientes con IAAS tuvieron un exceso de 16 días de estancia respecto a los no infectados. 
Tabla 2. Comparación de desenlaces en pacientes con y sin IAAS. Hospital Universitario Clínica San Rafael, Bogotá, Colombia

\begin{tabular}{|c|c|c|c|}
\hline & Pacientes infectados ( $\mathrm{n:}$ 187) & Pacientes no infectados (n: 276) & $\mathbf{p}$ \\
\hline Costos de hospitalización total US\$ (mediana y IC 95\%) & $6.329,1(5.526,9-7.933,7)$ & $1.206,7(973,6-1.495,0)$ & 0,001 \\
\hline Mortalidad & $59 \quad(31,55 \%)$ & $14 \quad(5,07 \%)$ & 0,001 \\
\hline Pacientes con hospitalización previa & $46 \quad(24,59 \%)$ & 0 & 0,001 \\
\hline Días de estancia hospitalaria (rango) & $21 \quad(18-24)$ & $5 \quad(5-6)$ & 0,001 \\
\hline Costo total de medicamentos generales US\$ (mediana e IC95\%) & $755,1(530,4-1.171,6)$ & $628,7(47,1-935,1)$ & 0,003 \\
\hline Costo total de antimicrobianos US\$ (mediana y IC 95\%) & $132,5(81,6-260,1)$ & $4,7(3,0-7,5)$ & 0,001 \\
\hline
\end{tabular}

Tabla 3. Comparación de costos (medianas e IC 95\% en US\$) de pisos y UCI1 de pacientes con y sin IAAS ${ }^{2}$. Hospital Universitario Clínica San Rafael, Bogotá, Colombia.

\begin{tabular}{lrl} 
& Pacientes infectados (n: 187) \\
Costo hospitalización en pisos & $4.368,12$ & $(3586,7-5036,6)$ \\
Costos fijos hospitalización en pisos & $2.205,6$ & $(1646,6-2706,2)$ \\
Costos variables hospitalización en pisos & $1840,5 \quad(1453,3-2183,8)$ \\
Costo día hospitalización en pisos & $270,9 \quad(229,7-315,4)$ \\
Costo total hospitalización en UCl & $5.928,0 \quad(4590,9-7532,6)$ \\
Costo día hospitalización en UCI & $566,6 \quad(507,5-691,3)$ \\
Costo de antimicrobianos en UCl & $45,5 \quad(33,4-94,6)$ \\
Costo de medicamentos generales en UCl & $400,8 \quad(288,2-486,7)$ \\
\hline
\end{tabular}

\begin{tabular}{cc} 
Pacientes no infectados $(\mathbf{n}: \mathbf{2 7 6})$ & $\mathbf{p}$ \\
$1.179 \quad(949,2-1331,8)$ & 0,002 \\
$567 \quad(446,9-726,4)$ & 0,001 \\
$456,1(376,5-571,8)$ & 0,001 \\
$193,6(174,5-228,8)$ & 0,05 \\
$637,2(459,5-691,3)$ & 0,001 \\
$312,9(219,4-423,6)$ & 0,05 \\
$3,0(1,5-5,8)$ & 0,001 \\
$47,7(38,5-71,3)$ & 0,02 \\
\hline
\end{tabular}

\section{Tabla 4. Costos (medianas e IC 95\% en US\$) atribuibles por tipo de IAAS. Hospital Universitario Clínica San Rafael, Bogotá, Colombia}

\begin{tabular}{|c|c|c|c|c|c|c|}
\hline Tipo de IAAS & $\begin{array}{c}\text { Costo infectados } \\
\text { US\$ }\end{array}$ & $\begin{array}{l}\text { IC } 95 \% \text { Costo } \\
\text { infectados US\$ }\end{array}$ & $\begin{array}{c}\text { Costo controles } \\
\text { US\$ }\end{array}$ & $\begin{array}{c}\text { IC } 95 \% \text { controles } \\
\text { US\$ }\end{array}$ & $\begin{array}{c}\text { Costo atribuible } \\
\text { de la IAAS US\$ }\end{array}$ & $\begin{array}{c}\text { IC } 95 \% \text { costo de } \\
\text { la IAAs }\end{array}$ \\
\hline Bacteriemia & 15.824 & $6.509-54.102$ & 513 & $215-4.558$ & 15.310 & $6.294-49.544$ \\
\hline Bacteriemia asociada a catéter & 32.272 & $13.894-29.102$ & 16.417 & $7.084-33.307$ & 15.855 & $6.810-45.956$ \\
\hline Endometritis & 370 & $263-835$ & 153 & $136-2.900$ & 217 & $126-2.064$ \\
\hline ISQ órgano espacio & 9.902 & $1.448-50.547$ & 1.914 & $1.808-4.960$ & 7.988 & $2.352-45.586$ \\
\hline ISQ profunda & 5.652 & $1.999-30.575$ & 345 & $244-445$ & 4.684 & $1.555-30.331$ \\
\hline IVU & 7.223 & $2.348-41.466$ & 1.650 & $286-7.649$ & 5.573 & $2.061-33.816$ \\
\hline Neumonía & 3.252 & $954-47.702$ & 2.041 & $226-5.196$ & 1.211 & $728-42.506$ \\
\hline
\end{tabular}

El requerimiento de hospitalización en UCI se presentó en $54,5 \%$ de los pacientes infectados, con un exceso de cinco días de estancia comparado con los pacientes sin IAAS que también requirieron UCI. La mortalidad en el grupo de pacientes infectados fue de $31,6 \%$ en comparación a los no infectados que fue de $5,1 \%$, con una mortalidad atribuible de $26,4 \%$

La mediana del costo total de hospitalización en los pacientes infectados fue US\$ 6.329 y la media US\$ 13.006, en comparación a los no infectados cuya mediana de costos fue US\$ 1.207 (19\% del valor de los infectados y la media US\$ 2.600 (41\% del valor de los infectados). La mediana del costo de antimicrobianos en pacientes infectados fue de US\$132,5 y la media US\$417, comparado con la mediana de los no infectados que fue US\$ 4,7 y la media US\$ $52(2,67 \%$ y $12 \%$ del costo de los antimicrobianos de los infectados respectivamente).

Dado que algunos pacientes presentaron más de una IAAS durante su hospitalización, se realizó un análisis separado según el número de infecciones para evitar confundir los resultados del costo atribuible a la IAAS. Los costos, la duración de la hospitalización, el requerimiento de hospitalización en UCI y la mortalidad fueron mayores en aquellos pacientes con tres IAAS. (Mediana de costos 
tres IAAS US\$ 23.088, media US\$ 27.710; costos de dos IAAS mediana US\$ 15.198,8 media US\$24.706; costos de una IAAS mediana US\$ 5.571,4, media US\$ 10.754).

En el análisis de costos por IAAS (evento individual), los costos atribuibles más altos se documentaron en bacteriemia asociada a catéter US\$15.855 (US\$ 6.810US\$ 45.956), bacteriemia US\$15.310 (US\$ 6.294US\$ 49.544) e ISQ órgano espacio US\$7.988 (US\$ 2.352US\$ 45.586) (Tabla 4).

\section{Discusión}

Los análisis de costos de las IAAS son complejos por la variabilidad en la relación de costos directos e indirectos originados en la atención en salud. No obstante, estudios como éste permiten tener una aproximación del impacto económico y social de este evento relacionado con la atención en salud. Nuestro estudio demuestra que las IAAS siguen siendo un problema serio, asociadas a exceso de días de hospitalización y uso de recursos de alta complejidad y a una mayor mortalidad. La metodología empleada, permitió controlar las principales variables que pudieran afectar el cálculo del costo de las IAAS como fue el control por gravedad de la enfermedad de base, edad, diagnósticos de ingreso y co-morbilidades.

La mortalidad atribuible a las IAAS en nuestro estudio fue de $26,4 \%$. Los estudios realizados para evaluar el impacto de las IAAS han encontrado datos variables respecto a la mortalidad atribuible según el tipo de IAAS y el microorganismo causal. El estudio realizado por M. Borges en Brasil, encontró una mortalidad atribuible de 45\% a las bacteriemias por Staphylococcus aureus ${ }^{8}$. Laupland K, en su estudio de bacteriemias en Canadá documentó una mortalidad atribuible de $16 \%{ }^{3}$. La mortalidad atribuible a bacteriemias por Acinetobacter baumannii multiresistente en el estudio de NY Lee fue de $21,8 \%{ }^{6}$. La mortalidad atribuible a candidemias fue de $24 \%{ }^{9}$. Los estudios realizados en neumonía asociada a ventilación mecánica (NAVM), documentaron una mortalidad atribuible variable, de $16 \%{ }^{10}$ y $7,3 \%{ }^{11}$. Respecto a las ISQ, un estudio que evaluó el impacto de la ISQ profunda en cirugía de fémur, no encontró una diferencia estadísticamente significativa en la mortalidad de los pacientes infectados respecto a los no infectados ${ }^{12}$, a diferencia del estudio de Coello R, que en su estudio de impacto de las ISQ en Inglaterra, sólo encontró como factor de riesgo para mortalidad la ISQ de prótesis de cadera ${ }^{2}$. El estudio de Coskun D, que evaluó ISQ en esternotomías, documentó una mortalidad de $19,2 \%$ en los pacientes con ISQ profunda respecto a los de ISQ superficial y no infectados ${ }^{13}$.

El exceso de costos atribuible a las IAAS en general fue de US\$ 5.122. Los costos atribuibles por tipo de infección fueron similares a los reportados por las UCI de Canadá (US\$ $12.321,20 \%$ mayor) ${ }^{3}$ y Bélgica (US\$ 17.836, 15\% menor $)^{14}$ en el caso de bacteriemias, menor a la reportada por bacteriemia por $S$. aureus (US\$ $82.818,81 \%$ menor) ${ }^{8}$, mayor a la reportada por bacteriemia por $A$. baumannii multiresistente (US\$ 3.758, 75\% mayor) ${ }^{6}$ y menor a la atribuible a NAVM (US\$ $11.897^{10}$, US\$ $11.100^{1}$, 90\% menor) posiblemente porque en el estudio este no fue el único tipo de neumonía incluido. Respecto al costo atribuible a ISQ, los costos fueron mayores que los reportados en la literatura (US\$ $2.200^{1}$, US\$ $3.740,58^{13}, 72 \%$ y $53 \%$ mayor, respectivamente). En el estudio el exceso de costos se presentó principalmente por los costos de la hospitalización en UCI y el consumo de antimicrobianos.

El exceso de días de estancia hospitalaria atribuible a IAAS fue de 16 días, la que fue menor a la encontrada en los estudios de ISQ esternal (21 y 35 días) ${ }^{13}$, NAVM $(25 \text { días })^{10}$, bacteriemia $\left(21\right.$ días $^{14}, 25$ días $\left.^{8}\right)$ e infecciones micóticas invasoras $\left(21\right.$ días $\left.^{15}\right)$.

El presente estudio, similar a algunos estudios observacionales, puede presentar factores de confusión no controlados debido a la recolección de datos de manera retrospectiva.

En conclusión, las IAAS se asocian con un exceso de costos, prolongación de estancia hospitalaria y mortalidad. Los resultados del estudio respecto al impacto de las IAAS son consistentes con lo publicado en otros países. Debe optimizarse las intervenciones dirigidas al control de las IAAS para garantizar una atención segura a los pacientes y mejorar el rendimiento de los recursos de los hospitales.

\section{Resumen}

Introducción: Las infecciones asociadas a la atención en salud (IAAS) están relacionadas con un incremento en los costos de hospitalización y un mayor riesgo de mortalidad. Objetivo: Establecer los costos y la mortalidad asociados a la presentación IAAS en una institución de cuarto nivel. Métodos: Se hizo una selección pareada de pacientes con IAAS y sin IAAS para calcular el costo por medio de costeo directo y emparejamiento. Los costos fueron calculados en pesos colombianos y convertidos a dólares estadounidenses según la tasa de cambio de 2008. Resultados: Se incluyeron 187 pacientes con IAAS y 276 pacientes sin IAAS. La tasa de IAAS fue de 1,8\% La mediana del costo de hospitalización en los pacientes con IAAS fue US\$ 6.329 (95\% CI US\$ 5.527-7.934) y en los no infectados de US\$1,207 (95\% CI US\$ 974-1.495). Los pacientes con IAAS presentaron mayor tiempo de estancia hospitalaria, con una diferencia de 16 días respecto a los no infectados (21 días (IC 95\% 18-24) vs 5 días (IC 95\% 5-6)). Se encontró una mortalidad atribuible de 26,4\%. Conclusiones: Las IAAS son eventos adversos a la atención, que se asocian con mayor mortalidad y generación de costos extra. 


\section{Referencias bibliográficas}

1.- Eber M R, Laxminarayan R, Perencevich E N, Malani A. Clinical and economic outcomes attributable to health care-associated sepsis and pneumonia. Arch Intern Med 2010; 170: 347-53.

2.- Coello R, Charlett a, Wilson J, Ward V, Pearson A, Borriello P. Adverse impact of surgical site infections in English hospitals. J Hosp Infect 2005; 60: 93-103.

3.- Laupland K B, Lee H, Gregson D B, Manns B J. Cost of intensive care unit-acquired bloodstream infections. J Hosp Infect 2006; 63: 124-32.

4.- Kollef K E, Schramm G E, Wills A R, Reichley R M, Micek S T, Kollef M H. Predictors of 30-day mortality and hospital costs in patients with ventilator-associated pneumonia attributed to potentially antibiotic-resistant gram-negative bacteria. Chest 2008; 134: 281-7.

5.- Saint S. Clinical and economic consequences of nosocomial catheter-related bacteriuria. Am J Infect Control 2000; 28: 68-75.

6.- Lee N-Y, Lee H-C, Ko N-Y, Chang C-M, Shih H-I, Wu C-J, et al. Clinical and economic impact of multidrug resistance in nosocomial
Acinetobacter baumannii bacteremia. Infect Control Hosp Epidemiol 2007; 28: 713-9.

7.- Horan T C, Andrus M, Dudeck M A. CDC/ NHSN surveillance definition of health careassociated infection and criteria for specific types of infections in the acute care setting. Am J Infect Control 2008; 36 (5): 309-32.

8.- Primo M G B, Guilarde A O, Martelli C M T, Batista L J D A, Turchi MD. Healthcareassociated Staphylococcus aureus bloodstream infection: length of stay, attributable mortality, and additional direct costs. Braz $\mathrm{J}$ Infect Dis 2012; 16: 503-9.

9.- Morgan J, Meltzer M I, Plikaytis B D, Andre N, White S H, Wilcox S, et al. Excess mortality, hospital stay, and cost due to candidemia: a case-control study using data from population -based candidemia surveillance. Infect Control Hosp Epidemiol 2005; 26 (6): 540-7.

10.- Warren D K, Shukla S J, Olsen M A, Kollef M H, Hollenbeak C S, Cox M J, et al. Outcome and attributable cost of ventilatorassociated pneumonia among intensive care unit patients in a suburban medical center. Crit Care Med 2003; 3: 1312-7.
11.- Hugonnet S, Eggimann P, Borst F, Maricot P, Chevrolet J C, Pittet D. Impact of ventilatorassociated pneumonia on resource utilization and patient outcome. Infect Control Hosp Epidemiol 2013; 25: 1090-6.

12.- Pollard T C B, Newman J E, Barlow N J, Price J D, Willett K M. Deep wound infection after proximal femoral fracture: consequences and costs. J Hosp Infect 2006; 63 : 133-9.

13.- Coskun D, Aytac J, Aydinli A, Bayer A. Mortality rate, length of stay and extra cost of sternal surgical site infections following coronary artery bypass grafting in a private medical centre in Turkey. J Hosp Infect 2005; 60: 176-9.

14.- Pirson M, Dramaix M, Struelens M, Riley T V, Leclercq P. Costs associated with hospital-acquired bacteraemia in a Belgian hospital. J Hosp Infect 2005; 59: 33-40.

15.- Menzin J, Lang K M, Friedman M, Dixon D, Marton J P, Wilson J. Excess mortality, length of stay, and costs associated with serious fungal infections among elderly cancer patients: findings from linked SEER-Medicare data. Value Health 2005; 8: 140-8. 